\title{
How to Catch a Massive Neutrino*
}

\author{
C. A. Heusch \\ PPE Division, CERN, Geneva, Switzerland \\ and \\ Institute for Particle Physics, University of California, Santa Cruz, California, U.S.A.
}

\begin{abstract}
In the absence of definitive information on either the exact values of light-neutrino masses or their Dirac or Majorana character, the existence of heavy neutrino states mixing with the light ones provides a viable overall scenario. We review available evidence and argue that the process $\mathrm{e}^{-} \mathrm{e}^{-} \rightarrow$ $\mathrm{W}^{-} \mathrm{W}^{-}$is likely to prove the preferred discovery channel for TeV-range Majorana masses that may explain the observed low-mass spectrum.
\end{abstract}

\section{INTRODUCTION}

Notwithstanding a great deal of theoretical as well as experimental effort on different fronts, the neutrino mass question has not seen a convincing breakthrough. We have three neutrino flavours in a framework of three quark/lepton families; but the rest masses accompanying these flavours, if not zero, are below our measurement sensitivity.

Any attempt at understanding our basic fermion spectrum in terms of some highersymmetry group will make us expect some compatibility between quark and lepton sectors, so that baryon number and lepton number can be seen as fundamental charges imposing some conservation scheme on particle interactions. For massless neutrinos, however, the concept of lepton number becomes meaningless: the apparent conservation of lepton number is simply due to helicity conservation, since

$$
\text { CPT }\left|v_{i}\right\rangle(\lambda) \rightarrow \eta\left|\bar{v}_{i}\right\rangle(-\lambda)
$$

a massless neutrino, which cannot change its chirality, will be prevented from producing a different 'lepton number' (say, $v_{e} \rightarrow e^{+}$) irrespective of any conservation law of this number. In fact, for $m(v)=0$, there is no distinction between our usual Dirac neutrino and a Majorana neutrino, which is an eigenstate of $C$ :

$$
c\left|v_{M}\right\rangle \rightarrow \eta_{M}\left|v_{M}\right\rangle
$$

The question of neutrino masses thus acquires another dimension: it permits us to probe for the very nature of neutral leptons. The more general concept is that of Majorana masses, which would set neutrinos apart from all other fundamental fermions, whereas it takes a special symmetry to establish Dirac masses which fit more easily into lepton-quark 'families'.

Is all of this a purely academic discussion in the absence of any compelling indication of non-zero neutrino rest masses? By no means: to establish a credible and plausible highersymmetry scheme encompassing both leptons and quarks, the vexing evidence of very small masses for the known neutrinos can be seen as an element in a scheme where charged leptons and quarks acquire their masses in the conventional way, to be compared with 'mixing masses' for the neutral leptons, as first introduced by Fritzsch and Minkowski [1], that have since been invoked in terms of the 'see-saw'

\footnotetext{
* Work supported in part by the U.S. Department of Energy.
} 
mechanism. In this context, the product of the masses of a light neutrino $(v)$ and a (conjectured) heavy one ( $N$ ) will be related to the typical fermion mass of a generation via

$\mathrm{m}_{\mathrm{v}} \cdot \mathrm{m}_{\mathrm{N}} \approx \mathrm{m}_{\mathrm{f}}^{2}$,

where $m_{f}$ can be a quark or a charged-lepton mass. Such heavy neutrino states $N$ occur naturally in a number of higher-symmetry schemes, notably in decompositions of the $\mathrm{E}(6) \rightarrow \mathrm{SO}(10) \rightarrow \ldots$ chain [2]. In other words, the existence of heavy neutrinos with masses $\geq 1 \mathrm{TeV}$, well above any limit that has been experimentally accessible, would tend to put the mass question for the 'light' neutrinos into the realm of easier accommodation in broad classes of models that contain our Standard Model as a low-energy phenomenology. The heavy masses satisfying Eq. 3 are naturally to be understood as Majorana masses [3].

\section{OBSERVABILITY OF MAJORANA MASSES}

Suppose now we want to verify the Majorana character of neutrino masses. How do we utilize the fact that the only observable difference between $|v\rangle$ and CPT $|v\rangle$ is the eigenvalue of the $\mathrm{T}$ operator (Eq. 1), i.e., the helicity? First of all, remember that for very small or vanishing neutrino masses, a helicity flip cannot be induced. Traditionally, the Tinvariance or noninvariance has been tested by

- detailed balance measurements or

- observation of T-violating decays.

Neither of these approaches is accessible to us for neutrinos. Nevertheless, the fact that $v_{M}, N$ exchanges may mediate a $\Delta \mathrm{L}=2$ transition points the way out of the 'practical Dirac/Majorana confusion' problem. The beststudied experimental approach to this problem is:

\section{A. Neutrinoless double $\beta$ decay}

This process, illustrated by the diagram in Fig. 1, is predicated on the likelihood that two d quarks inside two different neutrons in an appropriate nucleus overlap so as to exchange a $v_{M}$, and that the resulting two $u$ quarks wind up in two different protons. The ensuing process

$(\mathrm{A}, \mathrm{Z}) \rightarrow 2 \mathrm{e}^{-}+(\mathrm{A}, \mathrm{Z}+2)$

will be mediated by an amplitude

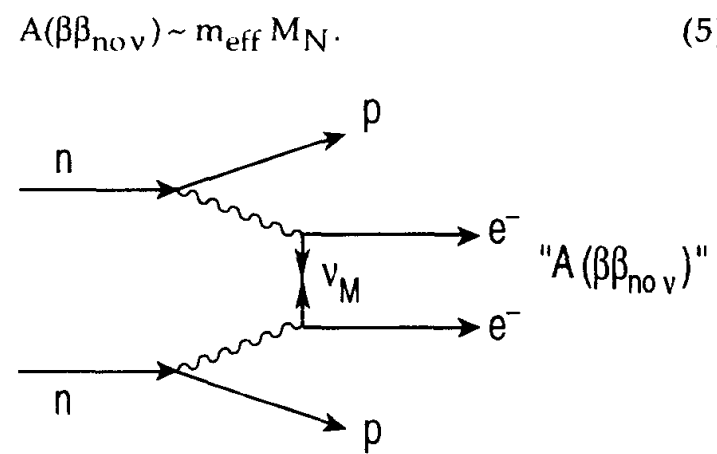

Figure 1: The dominant graph expected in neutrinoless double beta decay $\beta \beta_{\mathrm{no}}$ v

Here, $M_{N}$ is the hard-to-evaluate nuclear matrix element and $m_{\text {eff }}$ is some effective neutrino mass term

$m_{e f f}=\left|\sum_{i} \alpha_{e i}\left[U_{e i}\right]^{2} m_{v_{M}}\right|$,

with $m_{v_{M}}$ the mass eigenstates of all exchanged neutrinos, and $\mathrm{U}_{\mathrm{ei}}$ a mixing matrix describing their coupling to electrons. In other words, the amplitude for $\left(\beta \beta_{\text {no }}\right.$ ) observation is proportional to the neutrino mass (for small masses) due to helicity suppression; for any observed $\beta \beta_{\text {no }} v$ decay lifetime, it leads to the conclusion that at least one neutrino must have a Majorana mass [4]

$\mathrm{m}_{v} \geq 1 \mathrm{eV} \sqrt{\frac{\tau_{\text {Nucleus }}}{\tau_{\text {observed }}}}$.

In Eq. (7), the numerator is due to a nuclear matrix element calculation, and the denominator comes straight from experiment. 
Example: If the ongoing search for $\beta \beta_{\text {no }}$ v decay of ${ }^{76} \mathrm{Ge}$ led to the observation of an event at the edge of decay's upper limit $(\tau>24 y)$, the coincidence that $\tau_{\mathrm{Ge}}=10^{24} \mathrm{y}$ would lead to the conclusion that there is at least one $v_{M}$ with mass $m\left(v_{M}\right) \geq 1 \mathrm{eV}$.

In principle, the $\beta \beta_{\text {no }}$ v decay would be sensitive also to the exchange of a heavy Majorana neutrino; but the much heightened demand on the overlap of the parent $d$ quarks makes for a sharp mass suppression $\sim \mathrm{m}(\mathrm{N})^{-2}$; the calculated nuclear matrix elements are very unreliable and, most of all, there is no way to tell an experimental effect due to a light neutrino from one caused by a heavy $N_{M}$ state: a whole series of systematic measurements on different nuclei would be needed to pin this question down.

We conclude that, for all their usefulness for the detection of small Majorana masses, $\beta \beta_{\text {no } ~}$ projects are not in the running for $N_{M}$ detection.

B. Electron (or muon) capture with positron $\left(\mu^{+}\right)$ emission

The processes

$\mathrm{e}^{-}+(\mathrm{Z}, \mathrm{A}) \rightarrow \mathrm{e}^{+}+(\mathrm{Z}-2, \mathrm{~A})$

$\mu^{-}+(\mathrm{Z}, \mathrm{A}) \rightarrow \mu^{+}+(\mathrm{Z}-2, \mathrm{~A})$

are intimately related to the $\left(\beta \beta_{\text {no }}\right.$ ) process above [5]. Experimentation on process (8a) has systematic limitations due to radiative effects, but is in principle sensitive, again, to light as well as heavy Majorana masses. The radiative effects are not what limits process (8b) rather, the binding energy of the incident $\mu^{-}$ into a ${ }^{1} \mathrm{~S}$ atomic state of the $(Z, A)$ nucleus restricts the choice of $(Z-2, A)$ nuclei from which the $\mu^{+}$is to be ejected to appropriately light ones. Missimer et al. [6] point out that only radioactive targets such as ${ }^{44} \mathrm{Ti},{ }^{72} \mathrm{Se}$ fulfill this requirement. An evaluation of the process

${ }^{44} \mathrm{Ti}\left(\mu^{-}, \mu^{+}\right){ }^{44} \mathrm{Ca}$ can then be done in terms of the $v_{M}$ exchange graph of Fig. 2 and appropriate extensions of the Standard Model. Again, both light and heavy intermediate neutrinos can contribute, but estimates in left-right symmetric models or R-parity breaking supersymmetric models put observability will beyond the capabilities of currently existing muon factories with $>10^{8}$ stopping muons per second.

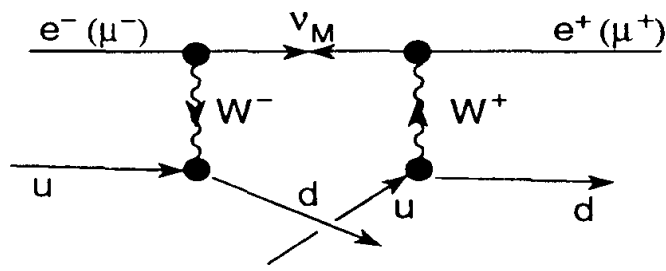

Figure 2: Electron (muon) capture with positron (antimuon) emission.

\section{Neutrino-antineutrino oscillations}

Another process where $\Delta \mathrm{L}=2$ transitions can be visualized is the so-called $v \bar{v}$ oscillation, shown schematically in Fig. 3 . From neutron $\beta$ decay, a reactor will emit large amounts of $\bar{v}_{e}$. If, in a detector at a sizeable distance from the reactor, the $\bar{v}_{e}$ flux produces, quasi-elastically, an electron instead of a positron, that will be a sure-fire sign that some $\bar{v}_{e}$ behave like $v_{e}$, i.e. it will be a signature for a Majorana mass.

Helicity suppression will again make this process occur, for light neutrinos, at a rate proportional to their mass; heavy Majorana masses will be hopelessly suppressed.

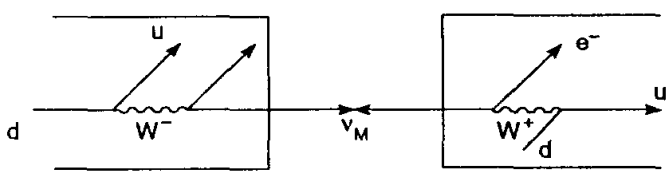

REACTOR

DETECTOR

Figure 3: Neutrino-antineutrino 'oscillation': neutron $\beta$ decay in a reactor emits an electron and a $\bar{v}_{e}$. A detector some distance away finds the $\bar{v}_{e}$ produces an electron, which is a product of $v_{e}\left(n o t \bar{v}_{e}\right)$ chargedcurrent scattering. 


\section{Muonium-antimuonium transitions}

For completeness, we mention that two $\Delta \mathrm{L}_{\mathrm{i}}=2$ transitions are the signatures for the process (see Fig. 4)

$\left(\mu^{+} \mathrm{e}^{-}\right) \rightarrow\left(\mu^{-} \mathrm{e}^{+}\right)$,

which has been investigated experimentally by $\mathrm{Ni}$ et al. [7]. Double helicity suppression makes this process, mediated by the exchange of two Majorana neutrinos, vulnerable at very small $\mathrm{m}\left(\mathrm{v}_{\mathrm{M}}\right)$; the suppression in the propagators for large masses is evident.

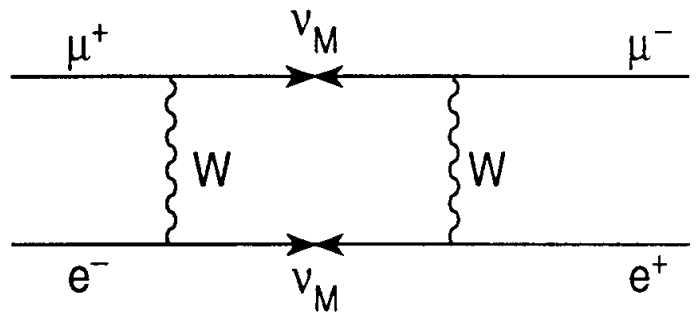

Figure 4: Muonium-antimuonium spontaneous conversion: the process is 'doubly forbidden' in the Standard Model. It takes a box diagram to characterize the lowest-order process.

\section{E. Production in high-energy ep scattering}

In electron-proton colliders such as HERA (or a possible LHC $\otimes L E P$ upgrade), a chargedcurrent interaction such as shown in Fig. 5 will be possible with a good experimental signature: the scattered charged lepton will emerge with the 'wrong' electric charge. The process

$\mathrm{e}^{-} \mathrm{p} \rightarrow \mathrm{v}_{\mathrm{M}} \mathrm{X}$

can lead to spectacular final-state signatures, such as $e^{+} \mu^{-}, e^{+} \tau^{-}$, or $e^{+} W^{-}-$where the $W^{-}$ can be well reconstructed from its hadronic decays. Detailed calculations [8,9] using the narrow-width approximation have shown that cross-sections are not hopelessly small, and that tight reconstruction cuts may now make the discovery of s-channel production and decay possible; however, as Fig. 6 shows explicitly, this discovery potential is limited to some $200 \mathrm{GeV}$ masses for HERA, to well below $1 \mathrm{TeV}$ even for the futuristic LHC $\otimes$ LEP scenario. In other words, we see no chance of penetrating to the $1-10 \mathrm{TeV}$ level indicated as the region of our primary interest, when using direct production from lepton beams.

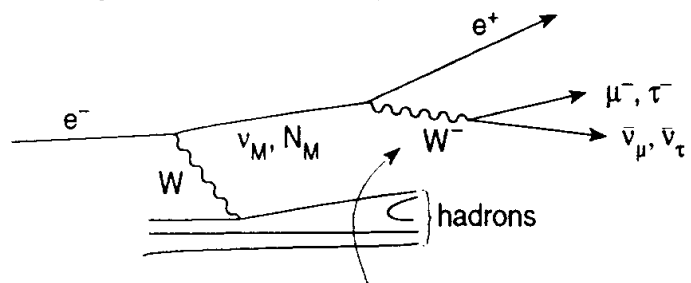

Figure 5: Deeply inelastic ep scattering: heavy $N_{M}$ states can result from charged-current interactions. The final-state pattern is spectacular.

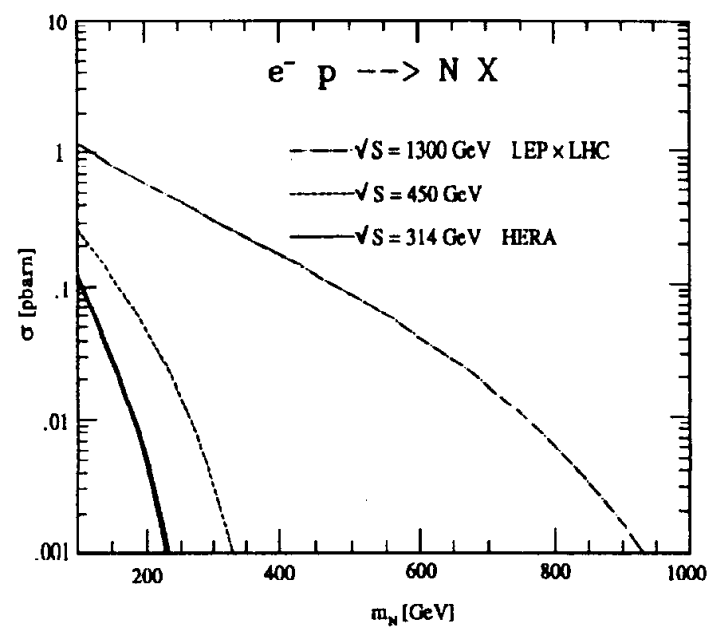

Figure 6: Cross-section for s-channel production of heavy $N$ states: the full line is for HERA and shows the inaccessibility of TeV-mass states. Broken lines indicate futuristic higher-energy scenarios.

\section{F. Production in $\mathrm{e}^{+} \mathrm{e}^{-}$colliders}

This also holds for neutrino production in the process

$\mathrm{e}^{+} \mathrm{e}^{-} \rightarrow \mathrm{N}_{\mathrm{M}} \mathrm{V}$

Buchmüller and Greub [8] considered this process for an NLC scenario, and give appropriate production distributions. For the mass range of our interest, however, there is no realistic discovery chance with foreseeable machines, so that we will, in the last section, concentrate on another option provided by the linear colliders for which process (11) was first evaluated. 


\section{MASSIVE NEUTRINOS IN $\mathrm{e}^{-} \mathrm{e}^{-}$ COLLISIONS}

Recent advances in linear accelerator technology as well as in the production and guidance of highly polarized electron beams of considerable intensity have opened up the possibility to revive precision experimentation with two incident electron beams [10], last performed over 30 years ago at centre-ofmass energies of a few hundred $\mathrm{MeV}$. Specifically, we maintain that the process

$\mathrm{e}^{-} \mathrm{e}^{-} \rightarrow \mathrm{W}^{-} \mathrm{W}^{-}$

has a fair chance of being the decisive discovery channel for heavy neutrinos. Heusch and Minkowski [11] have recently evaluated the cross-sections for a broad parameter space, after previous work had concentrated mostly on the framework of left-right symmetric theories [12-14].

Our motivation is this: if cross-sections are promising, the above channel (see Fig. 7) shows some unique advantages:

- there are plentiful electron sources available;

- high degrees of polarization can be reached easily, thus putting the choice of chiral couplings into our hands;

- easy back-to-back final-state kinematics will repress backgrounds efficiently;

- there are spectacular decay channels for the $\mathrm{W}^{-} \mathrm{W}^{-}$system, such as

$$
\begin{aligned}
\mathrm{e}^{-} \mathrm{e}^{-} \rightarrow \mathrm{W}^{-} \mathrm{W}^{-} & \rightarrow \mathrm{e}^{-} \mu^{-}\left(\tau^{-}\right)+\mathrm{p}_{\perp \text { miss }}, \\
& \rightarrow \mu^{-} \mu^{-}\left(\tau^{-}\right)+\mathrm{P}_{\perp \text { miss }} . \\
& \rightarrow \tau^{-} \tau^{-}+\mathrm{p}_{\perp \mathrm{miss}}, \\
& \rightarrow \mathrm{e}^{-}\left(\mu^{-}, \tau^{-}\right)+\text {jet }, \\
& \rightarrow \text { jet }+ \text { jet } .
\end{aligned}
$$
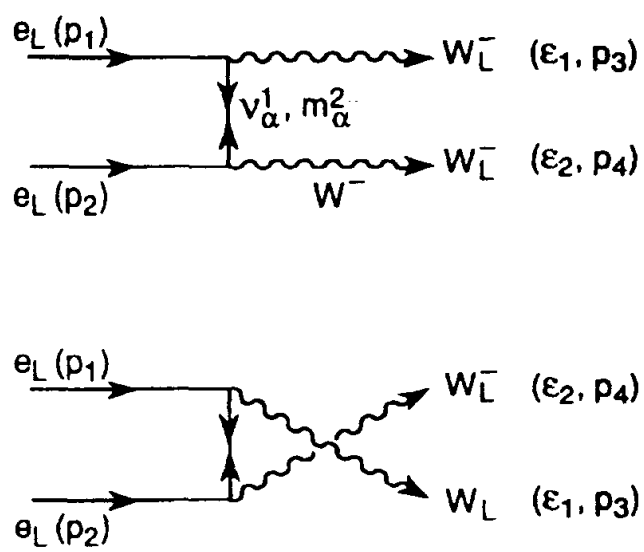

Figure 7: The 'elastic' process $\mathrm{e}^{-} \mathrm{e}^{-} \rightarrow \mathrm{W}_{\mathrm{L}}^{-} \mathrm{W}_{\mathrm{L}^{-}}^{-}$ where the couplings are of the kind $g F U_{e \alpha}$ and $U_{e \alpha}$ is a neutrino mixing matrix.

These signatures stand out from practically all reactions of the Standard Model and its likely extensions.

By investigating all viable helicity combinations for incoming electrons and outgoing $W_{\bar{L}}^{-}$bosons, we found that, in the notation of Fig. 7, the largest contribution to the cross-section for $\mathrm{W}_{\mathrm{L}}^{-} \mathrm{W}_{\mathrm{L}}^{-}$production is from the scattering of two left-handed electrons into a pair of longitudinally polarized ('scalar') $\mathrm{W}_{\mathrm{L}}^{-}$. The couplings in Fig. 7 show the mixing matrices $U_{e x}$ that are responsible for the mixing of light (e) and heavy neutrino flavours.

The resulting cross-section is [11], for exchange of a heavy Majorana neutrino $\mathrm{N}$,

$\sigma^{(N)}=\frac{G_{F}^{2} s}{16 \pi}\left(\frac{s}{m_{\text {red }}}\right)\left|\eta^{(N)}\right|^{2}$.

In Eq. 14, $\mathrm{m}_{\text {red }}$ stands for the heavy neutrino masses

$$
\frac{1}{m_{\text {red }}}=\sum_{\alpha} \frac{1}{m_{\alpha}},
$$


and we take it to be $m_{r e d} \approx 1 \mathrm{TeV}$, for calculational ease. $\eta^{(N)}$ is the neutrino mixing parameter

$$
\eta^{(\mathrm{N})}=\sum_{\alpha}\left(\mathrm{U}_{\mathrm{e} \alpha}\right)^{2} \frac{\mathrm{m}_{\mathrm{red}}}{\mathrm{m}_{\alpha}}
$$

which has to be culled from existing evidence on limits of lepton flavour violation and lepton flavour universality. We find, from the available evidence,

$$
\left|\eta^{(\mathrm{N})}\right| \leq(2-50) \times 10^{-4}
$$

The cross-section Eq. 14 is valid in the regime

$\mathrm{m}(\mathrm{W}) \leq \sqrt{\mathrm{s}} \leq 2 \mathrm{~m}_{\text {red }}$

but should be reasonably good up to $2 \mathrm{~m}_{\text {red. }}$. Its most prominent feature is $\sigma \sim s^{2}$, its strong increase with energy. Numerically, for $\mathrm{m}_{\text {red }}=$ $1 \mathrm{TeV}$, we then have

$$
\sigma^{(\mathrm{N})}=1 \mathrm{fb}\left(\frac{\sqrt{\mathrm{s}}}{0.5 \mathrm{TeV}}\right)^{4}\left|10^{3} \frac{\eta^{(N)}}{2}\right|^{2} .
$$

This cross-section is plotted for the upper and lower limits on $\eta^{(N)}$, in Fig. 8. For ' $N L C^{\prime}\left(\sqrt{s}_{s}=\right.$ $0.5 \mathrm{TeV}$ ) luminosities of $10^{33}-10^{34} \mathrm{~cm}^{-2} \mathrm{~s}^{-1}$, this leads to marginally acceptable event rates, for 'TLC' $(\sqrt{\mathrm{s}}=1 \mathrm{TeV})$ scenarios with $\mathscr{Y}=$ $10^{34}-10^{35} \mathrm{~cm}^{-2} \mathrm{~s}^{-1}$ to healthy counting rates.

Standard Model physics will, of course, populate all detector elements in competition with the possibly marginal signal. It is therefore of the greatest importance to know this competition in some detail. Two thorough recent studies $[15,16]$ have looked into all the processes within the Standard Model that will have two $\mathrm{W}^{-}$in the final state, together with two escaping neutrinos. Their unobserved $\mathrm{p} \perp$ and energy permit severe cuts on the total energy seen in the calorimeter, on the correlation angles of leptons and jets, and on missing transverse momentum observed in a hermetic detector, so that detection even of a few events should not present a serious problem: the tight kinematical constraints of back-to-back $W^{-}$emission in process (12) will permit its detection with a high degree of confidence.

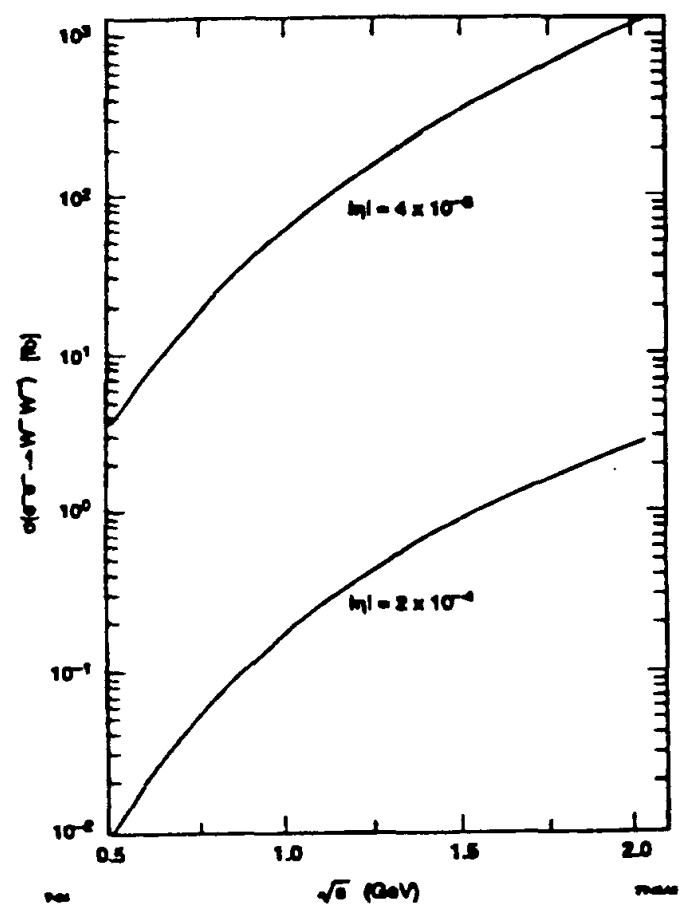

Figure 8 : Energy dependence of the cross-section for the process $\mathrm{e}^{-} \mathrm{e}^{-} \rightarrow \mathrm{W}_{\mathrm{L}}^{-} \mathrm{W}_{\mathrm{L}}^{-}$in the energy range of an NLC-type machine of a typical luminosity 10-100 $\mathrm{fb}^{-1}$, for the range of neutrino mass matrix choices discussed in Ref. [11]

\section{CONCLUSION}

It is our contention that the elastic production of two back-to-back $W^{-}$bosons is a promising method for the discovery of massive neutrinos with Majorana mass.

Higher-symmetry schemes appear to favour the existence of such neutral leptons in the $\mathrm{TeV}$ mass range. We do not know of another method for direct or indirect observation of their existence - which would help the 
understanding of the very light masses of the three known neutrinos [17]. In particular, the search for neutrinoless double $\beta$ decay, no doubt the most promising method for the discovery of light Majorana masses, is unlikely to be able to permit a search that can pin down a heavy neutrino mass. The method suggested here, moreover, is the only one where an experimental indication of heavy Majorana masses, due to its characteristic energy dependence, cannot be confused with another lepton number violating process, the exchange of doubly charged Higgs bosons [18].

This discovery potential of a crucial candidate mechanism for our understanding of the hitherto completely mysterious neutral lepton spectrum within our otherwise so attractive Standard Model phenomenology should give an electron-electron option high priority at all high-energy electron collider projects under development for the foreseeable future.

\section{REFERENCES}

1. H. Fritzsch and P. Minkowski, Phys. Lett. B62 (1976) 71.

2. P. Minkowski, Bern Preprint BUTP90/20 (1993).

3. B. Kayser, The Physics of Heavy Neutrinos, World Scientific, Singapore, 1989.

4. W.C. Haxton and G.J. Stephenson, Progr. Nucl. Part. Phys. 12 (1984) 409.

5. H. Primakoff and S.P. Rosen, Ann. Rev. Nucl. Part. Sci. 31 (1981) 145.

6. J.H. Missimer et al., PSI preprint PSIPR-94-09 (1994).

7. A. Halprin, Phys. Rev. Lett. 48 (1982) 1313;

B. Ni et al., Phys. Rev. Lett. 59 (1987) 2716.

8. W. Buchmüller and C. Greub, Nucl. Phys. B381 (1992) 109; and DESY 92-123 (1992).

9. G. Ingelman and J. Rathsman, DESY 93039 (1993).
10. C.A. Heusch, Proc. Workshop on Experimental Use of Linear $\mathrm{e}^{+} \mathrm{e}^{-}$ Colliders, F. Harris ed., World Scientific, Singapore, 1993, p. 895.

11. C.A. Heusch and P. Minkowski, Nud. Phys. B416 (1994) 3.

12. T. Rizzo, Phys. Lett. B116 (1982) 23; Proc. Workshop on Experimental Use of Linear $\mathrm{e}^{+} \mathrm{e}^{-}$Colliders, F. Harris ed., World Scientific, Singapore, 1993.

13. D. London, G. Belanger and J.N. Ng, Phys. Lett. B188 (1987) 155.

14. J. Maalampi, A. Pietilä and J. Vuori, Phys. Lett. B297 (1992) 327.

15. F. Cuypers et al., Phys. Lett. B325 (1994) 243.

16. V. Barger et al., MAD/PH/779 (1994).

17. S.M. Bilenky and C. Giunti: [P.L. B300, 137 (1993)] show that heavy neutrino admixtures could also cause a difference in $v_{\mu} \rightarrow v_{\tau}$ versus $\vec{v}_{\mu} \rightarrow \vec{v}_{\tau}$ transition probabilities, should they prove observable. We thank Prof. Bilenky for bringing this point to our attention after completion of the present manuscript.

18. R.N. Mohapatra and J.D. Vergados, Phys. Rev. Lett. 47 (1981) 1713. 
